\title{
Vaccine delivery system for tuberculosis based on nano-sized hepatitis B virus core protein particles
}

\author{
This article was published in the following Dove Press journal: \\ International Journal of Nanomedicine \\ 2 March 2013 \\ Number of times this article has been viewed
}

\section{Dhananjayan Dhanasooraj \\ R Ajay Kumar \\ Sathish Mundayoor}

Mycobacterium Research Group, Rajiv Gandhi Centre for Biotechnology,

Kerala, India
Correspondence: Sathish Mundayoor Mycobacterium Research Group, Rajiv Gandhi Centre for Biotechnology,

Thiruvananthapuram 695014 ,

Kerala, India

$\mathrm{Tel}+9|47| 2529512$

Fax +9| 47। 2348096

Emailsmundayoor@rgcb.res.in

\begin{abstract}
Nano-sized hepatitis B virus core virus-like particles (HBc-VLP) are suitable for uptake by antigen-presenting cells. Mycobacterium tuberculosis antigen culture filtrate protein 10 (CFP-10) is an important vaccine candidate against tuberculosis. The purified antigen shows low immune response without adjuvant and tends to have low protective efficacy. The present study is based on the assumption that expression of these proteins on $\mathrm{HBc}$ nanoparticles would provide higher protection when compared to the native antigen alone. The $c f p-10$ gene was expressed as a fusion on the major immunodominant region of HBc-VLP, and the immune response in Balb/c mice was studied and compared to pure proteins, a mixture of antigens, and fusion protein-VLP, all without using any adjuvant. The humoral, cytokine, and splenocyte cell proliferation responses suggested that the HBc-VLP bearing CFP-10 generated an antigenspecific immune response in a Th1-dependent manner. By virtue of its self-adjuvant nature and ability to form nano-sized particles, HBc-VLPs are an excellent vaccine delivery system for use with subunit protein antigens identified in the course of recent vaccine research.
\end{abstract}

Keywords: Mycobacterium tuberculosis, VLP, hepatitis B virus core particle, CFP-10, self-adjuvant, vaccine delivery

The number of new cases of tuberculosis (TB) continues to increase and approached 10 million globally in 2010. ${ }^{1}$ Bacille Calmette-Guerin (BCG) vaccination has long been considered as the best known form of disease prevention for tuberculosis, but unfortunately its efficacy varies from $0 \%$ to $80 \% .{ }^{2}$ Clinical trials of BCG have shown an almost total lack of protection in regions of the world where the disease is widespread, and thus BCG vaccination can be considered ineffective. ${ }^{3}$ In addition, BCG is a live vaccine and can cause disseminated disease in immunocompromised individuals. ${ }^{4}$ Therefore, there is an urgent need to develop more effective vaccines against TB.

Vaccines based on minimal pathogen components that are able to stimulate a protective response in a host are called subunit vaccines. Adjuvant-mediated vaccines are more stable and safer than classical vaccines, but without adjuvants these vaccines are less effective. ${ }^{5}$ Use of subunit vaccine with adjuvants is a promising approach for developing new vaccines against TB as well. In the case of TB, identification of Mycobacterium tuberculosis (Mtb) antigens recognized by both CD4 and CD8 T cells, which are capable of generating immune responses and blocking initial infection as well as reactivation, would be a good vaccine candidate. ${ }^{6}$ The proteins secreted by Mtb have been suggested for the protective immune response of live vaccines, ${ }^{7,8}$ so a vaccine based on these proteins is a promising strategy for an effective vaccine against TB. ${ }^{9}$ Subunit vaccination based on the secretory proteome of Mtb is a promising vaccine 
strategy against TB. ${ }^{10}$ Although a number of antigens with vaccine potential have been identified, the majority of vaccine studies have relied on a small number of immunodominant antigens from Mtb, of which one of the most studied is culture filtrate protein 10 (CFP-10). CFP-10 is strongly recognized as a T-cell antigen in the initial stage of infection and has well-characterized epitopes providing significant efficacy in animal models. ${ }^{11-13}$ CFP-10 induces Cytotoxic T lymphocyte (CTL) activity and interferon-gamma (IFN- $\gamma$ ) production in animal models and in humans infected with Mtb, making it an excellent anti-TB vaccine candidate. ${ }^{14,15}$

A particulate vaccine delivery system, such as nanosized virus-like particles (VLPs), can act as adjuvant as well as antigen delivery through enhancing the antigen uptake by antigen-presenting cells. ${ }^{16}$ VLPs also have a special advantage over a conventional system in size, stability, and capacity to transport across biological barriers. ${ }^{17}$ Particles in the 20-200 $\mathrm{nm}$ range are also able to generate CD4, CD8, and Th1 responses. ${ }^{18}$ Hepatitis B virus core protein-VLPs (HBc-VLPs) are in this size range and are able to generate both cellular and humoral immune responses. ${ }^{19-21}$

Recent research has shown that in comparison to native antigens, the fusion of two or more proteins when administered with adjuvants generated protective immunity in animal models. ${ }^{22}$ However, purified proteins show low immune response without adjuvants and tend to have a protective efficacy that is lower than BCG. CFP-10 is a major secretory protein and is one of the most immunodominant and pathogen-specific antigens from $\mathrm{Mtb}{ }^{23} \mathrm{~A}$ few studies have shown that nanoparticle-mediated delivery of immunogens may result in improved protection against TB. ${ }^{24-26}$ In this study we synthesized nano-sized HBc-VLPs bearing mycobacterial antigen CFP-10 and compared its immune response in a mice model. We also demonstrated that HBc-VLP::CFP-10 fusion protein induces an increased immune response in $\mathrm{Balb} / \mathrm{c}$ mice compared to mixtures of native antigen.

\section{Materials and methods Gene amplification and plasmid construction}

The gene sequence required for the formation of HBc-VLP (149 amino acid coding region) was amplified from genomic DNA of hepatitis B virus by using primers CEFP2 and CEHsR (designed to support a 6 HIS- tag at the C terminal of the expressed protein). The sequence of the HBc gene encoding major immunodominant region (MIR) was modified using overlap extension polymerase chain reaction (OEPCR) with primers CMPRP and CMPFP (Table 1).
The resulting MIR sequence formed restriction sites for Eco RI and Hind III, which were flanked by two linker sequences $\left(\mathrm{G}_{4} \mathrm{SG}_{4} \mathrm{~T}\right.$ and $\left.\mathrm{G}_{4} \mathrm{SG}_{4}\right)$ that replaced six nucleotides from the MIR region. The modified and unmodified $\mathrm{HBc}$ genes were separately cloned into pET32a by using Nde I and Xho I restriction sites, resulting in $\mathrm{pETMHBc}$ and $\mathrm{pETHBc}$ plasmids, respectively.

For cloning into $\mathrm{pETMHBc}$, the CFP-10 coding sequence was amplified from Mtb H37Rv by using primers Mcfp10F and Mcfp10R, which had restriction sites Eco RI and Hind III. The complete CFP-10 gene was amplified from Mtb H37Rv by using primers CFP10ptF and CFP10ptR (designed to sport a 6 HIS- tag at the $\mathrm{N}$ terminal of the expressed protein) containing restriction sites Nde I and Xho I and was cloned into $\mathrm{pET} 32 \mathrm{a}$ for expression and purification of CFP-10 (pETCFP).

\section{Protein expression and purification CFP-IO expression and purification}

For CFP-10 expression, pETCFP was transformed into Escherichia coli BL21(DE3) (Novagen, Billerica, MA, USA). Protein expression was induced by adding isopropyl1-thio- $\beta$-D-galactopyranoside (IPTG) at a final concentration of $0.5 \mathrm{mM}$ at $28^{\circ} \mathrm{C}$. The cell pellets were resuspended in lysis buffer (50 mM NaHPO $, 200 \mathrm{mM} \mathrm{NaCl}, \mathrm{pH} 8$ ), ultrasonicated, and centrifuged at 10,000 $\mathrm{g}$ for 20 minutes at $4^{\circ} \mathrm{C}$. The clear supernatant was collected, and the protein was purified by immobilized metal affinity chromatography with Ni-silica resin (Promega, Madison, WI, USA). Protein-bound resin was washed with buffer containing $20 \mathrm{mM}$ imidazole, and the bound protein was eluted with buffer containing $250 \mathrm{mM}$ imidazole. The eluted protein was desalted and concentrated with a U-tube concentrator (Novagen). Protein concentration was estimated by using the BCA assay (Thermo Scientific, Rockford, IL, USA), analyzed on sodium dodecyl sulfate-polyacrylamide gel electrophoresis and confirmed by Western blot using anti-CFP-10 antibody (Thermo Scientific). Lipopolysaccharide (LPS) contamination was determined using the EndoLISA endotoxin detection assay (Hyglos GmbH, Bavaria, Germany).

\section{Purification of VLPs}

Plasmids $\mathrm{pETHBc}$ and $\mathrm{pETMHBc}: \mathrm{CFP}-10$ were transformed into $E$. coli BL21 (DE3), and protein expression was induced with $0.5 \mathrm{mM}$ and $1 \mathrm{mM}$ IPTG, respectively. Purification of HBc-VLPs and fusion protein VLPs (FVLP) was performed as noted in earlier reports ${ }^{27,28}$ with minor modifications. Briefly, C-terminal HIS-Tagged proteins were purified using 
Table I Primer sequences

\begin{tabular}{lll}
\hline SI no & Primer name & Sequence \\
\hline 1 & CEFP2 & GGAATTCCATATGGACATTGACCCTTATAAAGA \\
2 & CEHsR & CCGCTCGAGCTAATGGTGATGGTGATGGTGAACAACAGTAGTCTCCGGAAGTG \\
3 & CMPRP & AAGCTTGGGCCGGAATTCGGTGCCACCGCCACCAGAGCCACCGCCACCATCTTCCAA \\
4 & CMPFP & GATTCCGGCCCAAGCTTGGTGGCGGTGGCTCTGGTGGCGGTGGCTCTAGGGAC \\
5 & McfpI0F & CCGGAATTCGCAGAGATGAAGACCGATG \\
6 & McfPIOR & GGAATTCCATATGCACCATCACCATCACCATGCAGAGATGAAGACCGATG \\
7 & CFPI0ptF & CCGCTCGAGTCAGAAGCCCATTTGCGAGGACAGC \\
8 & CFPI0ptR &
\end{tabular}

Notes: Sequences I and 2 are for amplification of the $\mathrm{HBc}$ gene for cloning into the PET vector. Sequences 3 and 4 are for modifying the MIR region (using overlap extension $P C R)$. Sequences 5 and 6 are for cloning the $c f p-10$ gene into the modified MIR region of the HBc gene. Sequences 7 and 8 are for cloning the $c f p-10$ gene into the $p E T$ vector.

Abbreviations: HBc, Hepatitis B virus core; PET, PET-32a; MIR, major immunodominant region; PCR, polymerase chain reaction.

$\mathrm{Ni}$-silica resin, and reassembled VLPs were recovered by $10 \%-60 \%$ sucrose density gradient ultracentrifugation. Proteins were detected by Western blotting using anti-HBc and anti-CFP-10 antibodies.

\section{Transmission electron microscopy}

Sucrose density gradient fractions were diluted in phosphatebuffered saline (PBS), layered onto copper grids, and negatively stained using $2 \%$ uranyl acetate. Electron micrographs were obtained on a transmission electron microscope (Jeol, Tokyo, Japan).

\section{Animals and immunization}

Pathogen-free Balb/c female mice (6-8-weeks old) were used for this study, and all experiments were approved by the Institutional Animal Ethics Committee. We used five groups of eight mice each: G1, sham immunized; G2, CFP-10 immunized; G3, HBc-VLP immunized; G4, $\mathrm{HBc}-\mathrm{VLP}$ and CFP-10 protein mixture (ratio similar to FVLPs) immunized; G5, FVLP immunized. Each group was immunized three times at 2-week intervals. One week after the final immunization, mice were sacrificed and blood and spleen were collected aseptically. The spleens were crushed and passed through a $70 \mu \mathrm{m}$ cell strainer (BD Falcon, Bedford, MA, USA). The red blood cells were lysed by using Ammonium-chloride-potassium (ACK) lysis buffer (150 mM $\mathrm{NH}_{4} \mathrm{Cl}, 10 \mathrm{mM} \mathrm{KHCO}, 0.1 \mathrm{mM}$ EDTA, $\mathrm{pH}$ 7.4) and then resuspended in warm RPMI (Sigma-Aldrich, St.Louis, MD, USA). The spleens were all processed separately.

\section{Antibody assay}

Direct enzyme-linked immunosorbent assay (ELISA) was used for detection of anti-CFP-10 antibodies in the sera of immunized animals. Briefly, ELISA plates (BD Falcon) coated with CFP-10 (5 $\mu \mathrm{g} /$ well $)$ in PBS overnight at $4^{\circ} \mathrm{C}$ were blocked with 3\% bovine serum albumin in PBS for 2 hours, washed with PBS containing $0.05 \%$ Tween 20 , and incubated with 1:500 prediluted serum. The washed plates were incubated with horseradish peroxidase-conjugated anti-mouse immunoglobulin (Ig)G (Sigma-Aldrich) or anti-mouse IgG1 or anti-mouse IgG2a (Santa Cruz Biotechnology, CA, USA). The plates were developed with tetramethylbenzidine substrate solution (BD Biosciences, San Jose, CA, USA) and $1 \mathrm{M} \mathrm{H}_{3} \mathrm{PO}_{4}$. The optical density (OD) at $450 \mathrm{~nm}$ was read on a microplate reader (Bio-Rad, Laboratories, Hercules, CA, USA).

\section{Cytokine analysis}

Splenocytes $\left(2 \times 10^{5}\right.$ cells/well $)$ were cultured in 96-well plates (BD Biosciences) in RPMI 1640 supplemented with $1 \%$ penicillin-streptomycin, $1 \mathrm{mM}$ glutamine, and $10 \%$ Fetal borine serum (FBS) at $37^{\circ} \mathrm{C} / 5 \% \mathrm{CO}_{2}$. Triplicate wells were stimulated with buffer (control), concanavalin A (Con A ), CFP-10, and culture filtrate (CF) of Mtb (prepared as per an earlier report $)^{29}$ at $5 \mu \mathrm{g} / \mathrm{mL}$. Supernatants were collected after 72 hours and IFN- $\gamma$, Interleukin (IL)-2, tumor necrosis factor (TNF), IL-5, and IL-10 were quantified using cytokine ELISA (BD Biosciences).

\section{Lymphocyte proliferation assay}

Splenocytes $\left(4 \times 10^{5}\right.$ cells/well $)$ were cultured in RPMI supplemented with $10 \%$ FBS and stimulated with CFP-10 $(5 \mu \mathrm{g} / \mathrm{mL})$ and Con A in triplicate. After 72 hours cell proliferation was assessed by using the CellTiter 96 kit (Promega) according to the manufacturer's instruction. The proliferative responses were expressed as the stimulation index (SI),

\section{ELISPOT assay}

Enzyme-linked immunospot (ELISPOT) assays were performed with the ELISPOT assay kit (BD Biosciences). 
Briefly, plates were coated with anti-mouse IFN- $\gamma$ antibody and blocked with RPMI, 10\% FBS, and L-glutamine. Splenocytes taken from individual mice were cultured in triplicate (at $5 \times 10^{5}$ cells/well) in RPMI for 20-24 hours at $37^{\circ} \mathrm{C}$ and $5 \% \mathrm{CO}_{2}$ with Con A or antigen (CFP-10). Cells were discarded post stimulation, and plates were developed with 3-amino-9-ethylcarbazole (AEC) substrate reagent (BD Biosciences). Spots were counted under a dissecting microscope (Olympus, Tokyo, Japan).

\section{Statistical analysis}

Statistical analysis was done by parametric testing of mean values via one-way analysis of variance (with Tukey's post hoc testing to identify between-group significance).

\section{Results}

\section{Expression of CFP-I0, $\mathrm{HBc}$, and FVLP}

Western blot using anti-CFP-10 and anti-HBc antibodies showed that all proteins were expressed correctly (Figure 1). Electron microscopic studies revealed that both $\mathrm{HBc}$ and fusion protein formed VLPs (Figure 2). The LPS levels in the purified proteins were found to be low at $\sim 1 \mathrm{ng} / \mathrm{mg}$ of antigen.

\section{FVLP generated a higher CFP-I0-specific humoral response}

To analyze whether the FVLP could enhance immunogenicity when compared to CFP-10 protein alone, ELISA

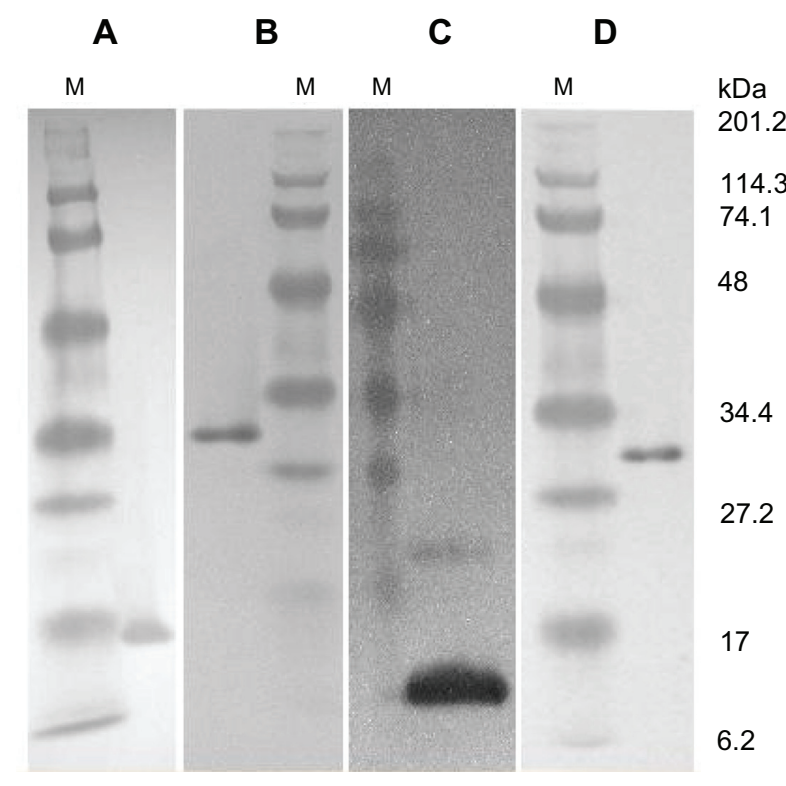

Figure I Expression of CFP-I0, HBc, and FVLP.

Notes: Western blot of purified proteins. A, purified $\mathrm{HBc}$ probed with anti-HBc antibody; B, purified FVLP with anti-HBc antibody; C, purified CFP- 10 with anti-CFP10 antibody; D, FVLP with anti-CFP-10 antibody.

Abbreviations: $M$, protein molecular weight marker in $\mathrm{kDa}$; CFP-10, Culture filtrate protein- 10; $\mathrm{HBc}$, Hepatitis B core protein. was performed to detect the levels of anti-CFP-10 antibodies in the sera collected from mice immunized with different proteins. The results showed that FVLP was more immunogenic and produced higher antibody responses than CFP-10 alone $(P<0.001)$ or CFP-10 mixed with $\mathrm{HBc}$ $(P<0.001)$ (Figure $3 \mathrm{~A})$. IgG1 production against FVLP was less than that of the other two groups (Figure $3 \mathrm{~B}$ ), although this was not statistically significant. However, significantly higher levels of IgG2a antibodies $(P<0.001)$ were produced against FVLP when compared to mixed proteins/CFP-10 (Figure 3C).

\section{Cytokine response}

Splenocytes from different groups of mice were stimulated with CFP-10, and cells from FVLP-immunized mice showed significantly higher production of IFN- $\gamma$, IL-2, and TNF compared to splenocytes from CFP-10 or mixture-immunized mice (Figure 4A-C). Interestingly, except for TNF the same picture emerged when they were stimulated with $\mathrm{CF}$. Stimulation of splenocytes from fusion protein-immunized mice with either CFP-10 or CF showed lower levels of both IL-5 and IL-10 (Figure 4D and E), although this was not statistically significant when compared to CFP-10 or mixtureimmunized mouse cells.

\section{FVLP-generated antigen-specific splenocyte proliferation}

Splenocytes from animals immunized with CFP-10 (G2) and FVLP (G5) produced significantly higher proliferative responses on stimulation with CFP-10 in vitro when compared to cells from the other three groups. Mice that were immunized with FVLPs demonstrated maximum proliferation of splenocytes against CFP-10 $(P<0.001)$ (Figure 5).

\section{FVLP-generated antigen-specific IFN- $\gamma$ secretion}

The number of IFN- $\gamma$-secreting cells was enumerated by ELISPOT assay. The cells from different groups of mice were stimulated with CFP-10. The FVLP group showed significantly $(P<0.001)$ higher numbers of IFN- $\gamma$-secreting cells compared to cells from CFP-10 alone or the protein mixture-immunized group of mice (Figure 6).

\section{Discussion}

CFP-10 is one of the ideal candidates for a subunit vaccine, either by itself or in combination with other relevant antigens of Mtb against TB. ${ }^{30}$ However, the specific immune response depends on the method of antigen delivery to the immune 
A

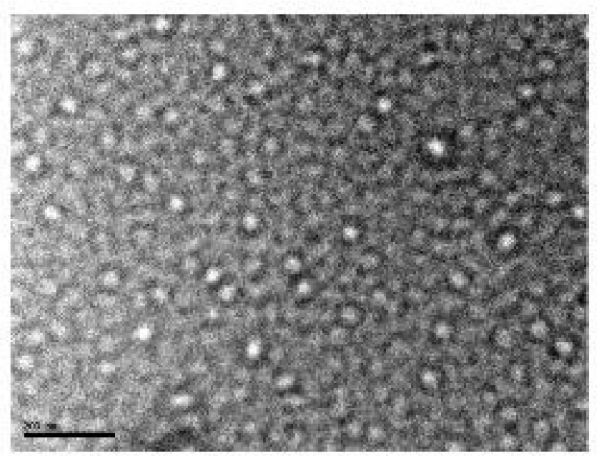

B

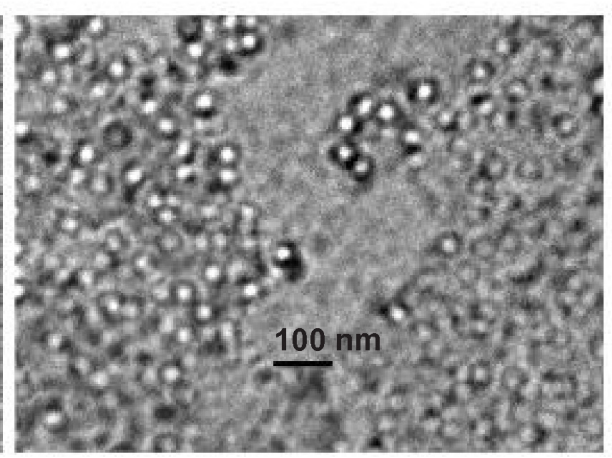

Figure 2 Transmission electron microscopic pictures of VLPs.

Notes: (A), HBc-VLP. Bar $=200 \mathrm{~nm}$; (B), FVLP. Bar $=100 \mathrm{~nm}$.

Abbreviations: VLPs, virus like particles; HBC-VLP, hepatitis B core protein VLP; FVLP, fusion protein VLP.

system. ${ }^{31}$ The critical component of protective immunity against TB is the T-cell-mediated response characterized by the secretion of IFN- $\gamma$ and other cytokines. ${ }^{32}$ Some recent studies have also suggested that antibodies also enhance the protective effect against TB. ${ }^{33,34}$ These reports strengthen the emerging view that the most effective immune response is the one that combines both humoral and cellular components. ${ }^{33}$

In this study, we expressed the full-length CFP-10 protein on HBc-VLP. Results from electron microscopy coupled with Western blots confirmed that the fusion protein was correctly expressed, was able to form VLPs, and that the presence of CFP-10 antigen did not affect VLP assembly. The immune responses generated by FVLP were analyzed after immunizing Balb/c mice with mixtures of separate antigens and as fusion VLPs. This also helped to analyze the self-adjuvant nature of HBc-VLPs. Our results showed that except for FVLP-immunized mice, other groups were not able to confer the same level of immune response. This strongly suggests that VLPs are an excellent carrier of Mtb antigens and could act as a self-adjuvant, an important factor for vaccination. The high antibody response to the FVLP-immunized group contrasts with the fact that simply mixing the CFP-10 protein in its native form with HBc-VLP was not able to generate similar antibody responses. Thus, the mere presence of CFP-10 protein and native HBc-VLP was not sufficient to generate strong immune responses, emphasizing that a proper display of foreign antigen on VLPs is essential. The high level of IgG2a response to FVLP was not achieved when the mice were immunized with mixtures of antigen. The significant elevation of anti-CFP-10 IgG2a in G5 mice indicates that a fusion protein carrier induces preferentially Th1-type priming and interaction, even though IgG1 (a marker of Th2 immune response) is usually the predominant isotype in Balb/c mice. ${ }^{35,36}$

IFN- $\gamma$ has been shown to be indispensable for resistance to mycobacterial and other intracellular infections and is often used as a single readout for Th1 responses..$^{22,37}$ The secretion of comparatively high amounts of IFN- $\gamma$ in response to CFP-
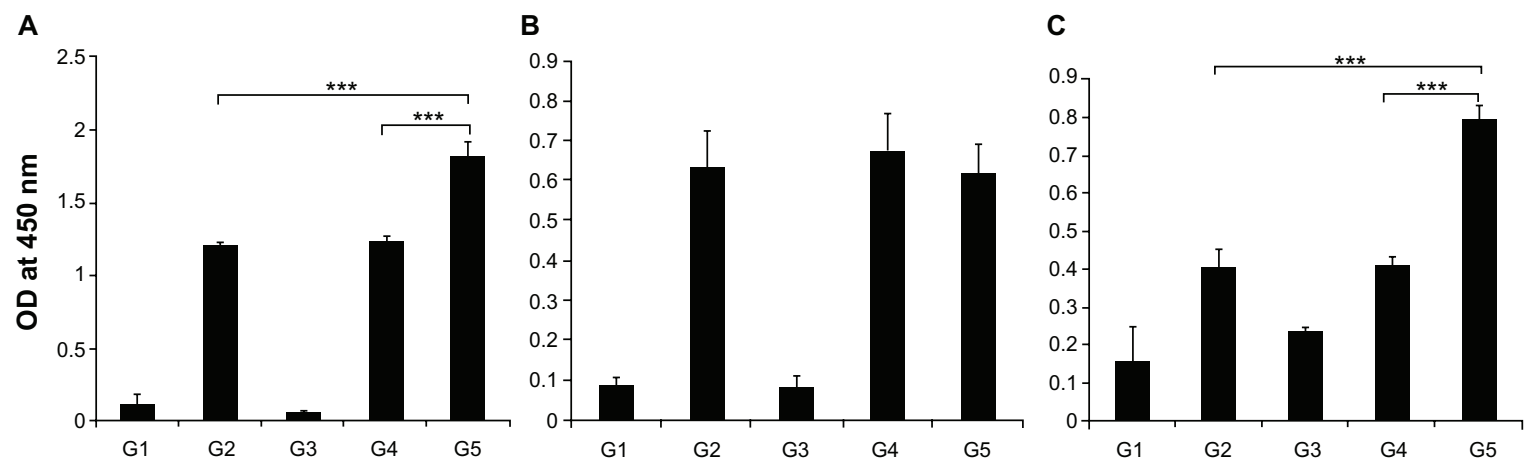

Figure 3 CFP-10 specific humoral responses in different mice groups after immunization. Blood samples from Balb/c mice were collected after final immunization. (A) Antibody response against CFP-10 in all groups. (B) CFP-I0-specific IgGI responses in immunized mice groups. (C) CFP-I0-specific IgG2a responses in immunized mice groups. Optical densities were read at $450 \mathrm{~nm}$, and results are expressed as the arithmetic mean of the titer obtained.

Notes: The data are presented as mean \pm standard deviation. Statistical significance between groups is indicated as $* * * P<0.00 \mathrm{I}$.

Abbreviations: OD, optical density; CFP-I0, culture filtrate protein-I0; GI, buffer-immunized group; G2, CFP-I0-immunized group; G3, HBc-VLP-immunized group; G4, $\mathrm{HBC}+\mathrm{CFP}-10$ mixture-immunized group; G5, FVLP-immunized group. 


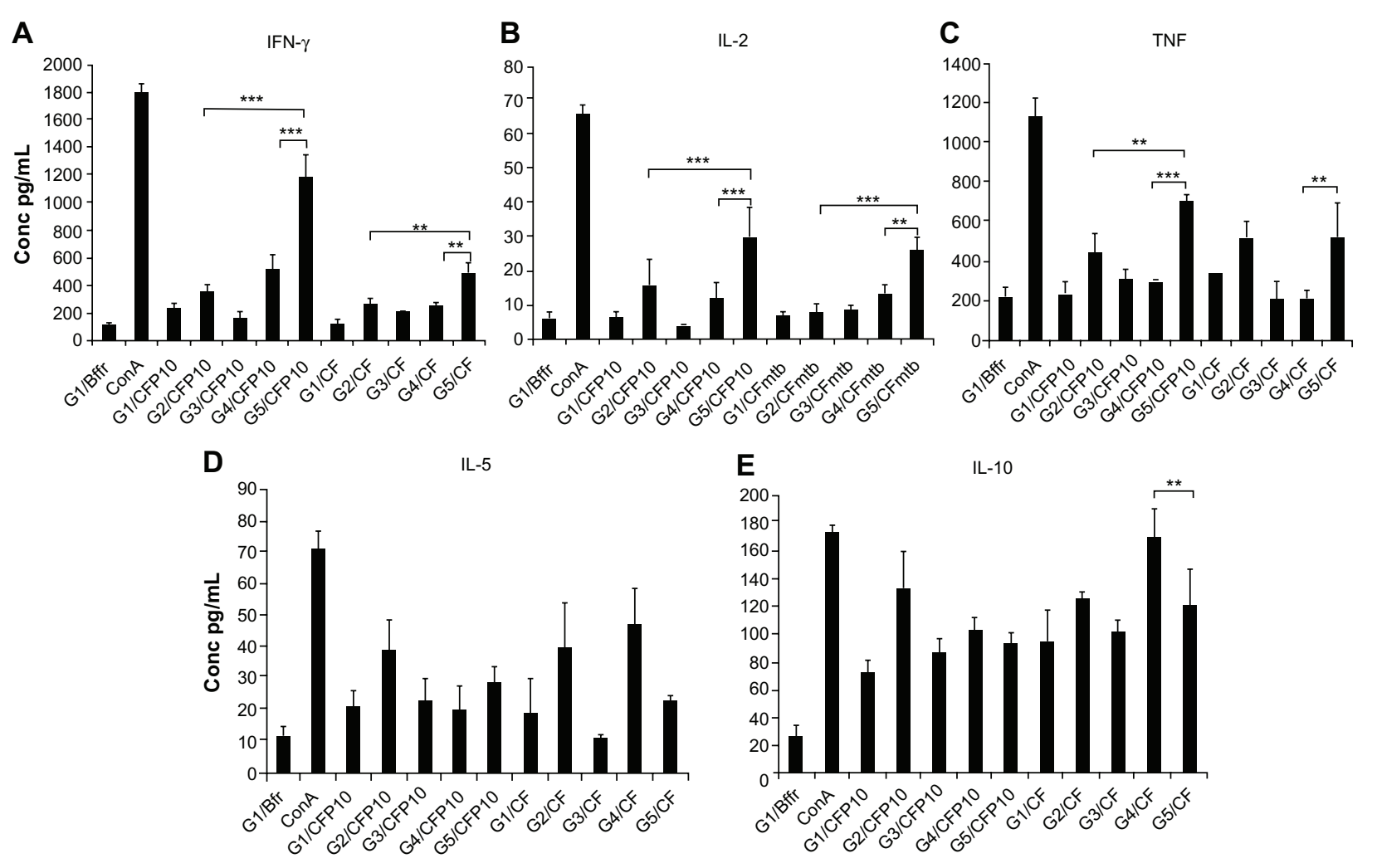

Figure 4 Cytokine production by the splenocytes stimulated with CFP-I0. Splenocytes collected from each group of mice I week after the final immunization were stimulated with CFP-10 $(5 \mu \mathrm{g} / \mathrm{mL})$. Con A $(5 \mu \mathrm{g} / \mathrm{mL})$ and buffer were used as controls. (A) IFN- $\gamma$; (B) IL-2; (C) TNF; (D) IL-5; (E) IL-I0.

Notes: The concentrations of cytokines in the splenocyte culture supernatant were determined by ELISAs. The data are presented as mean \pm standard deviation. Statistical significance between groups is indicated as $* * * P<0.001$, $* * P<0.05$

Abbreviations: IFN-V, interferon-gamma; IL-2, interleukin-2; TNF, tumor necrosis factor; IL-5, interleukin-5; IL-I0, interleukin-I0; CF, culture filtrate of Mtb; GI, bufferimmunized group; G2, CFP-I0-immunized group; G3, HBc-VLP-immunized group; G4, HBc + CFP-I0-immunized group; G5, FVLP-immunized group; Con A, concanavalin A; CF, culture filtrate of Mtb.

10 stimulation in splenocytes of mice immunized with FVLP is a further indication of proper antigen delivery by VLPs. In addition, these splenocytes also showed significant induction of IFN- $\gamma$ on stimulation with CF of Mtb. This was interesting because the secretion of $\mathrm{CF}$ proteins in experimental conditions resembles an environment that Mtb encounters in the host cell. In the absence of an infection model in this study, this immune response to CF can be considered as a protective response against $\mathrm{Mtb}$ challenge. IL-2 plays a major role in activation and proliferation of cytotoxic $\left(\mathrm{CD}^{+}\right) \mathrm{T}$ cells that develop later in the acquired immune response. ${ }^{38-40}$ Studies have also suggested a significant role for $\mathrm{CD} 8^{+}$cells in protective immune responses against $\mathrm{Mtb}^{41,42}$ The increased levels of IL-2 against CFP-10 and CF of Mtb also suggest a tilting of immune response in a Th1-dependent manner. TNF on its own is another effector cytokine that can synergize with IFN- $\gamma$ to eliminate intracellular pathogens. ${ }^{22,43,44}$ The higher secretion of TNF in G5 upon stimulation with CFP-10 points to the fact that FVLP was able to induce a protective type of immune response. Compared to all other mice groups, the minimal production of IL-5 and IL-10 in G5 on stimulation

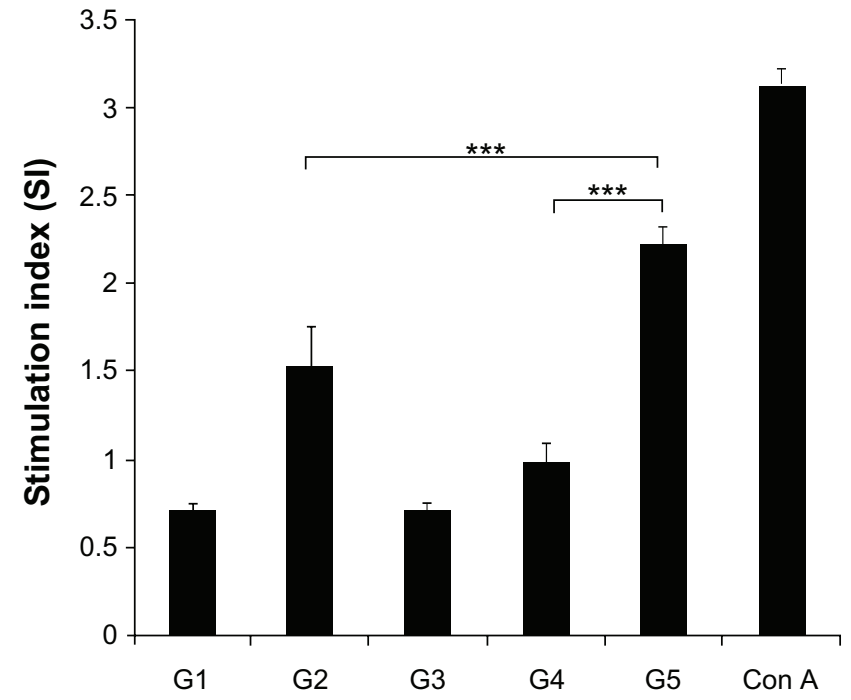

Figure 5 Effect of FVLP immunization on splenocyte proliferation.

Notes: Lymphocyte proliferation in response to CFP-10 $(5 \mu \mathrm{g} / \mathrm{mL})$ stimulation was evaluated using the CellTiter Cell Proliferation assay. Absorbance was measured at $490 \mathrm{~nm}$, and the proliferative responses were expressed as the stimulation index (SI). Con A $(5 \mu \mathrm{g} / \mathrm{mL})$ was used as control. The data are presented as mean \pm standard deviation. Statistical significance between groups is indicated as $* * * P<0.00$ I.

Abbreviations: CFP-10, culture filtrate protein-10; GI, buffer-immunized group; G2, CFP-I0-immunized group; G3, HBc-VLP-immunized group; G4, HBc + CFP-I0immunized group; G5, FVLP-immunized group; Con A, concanavalin A. 


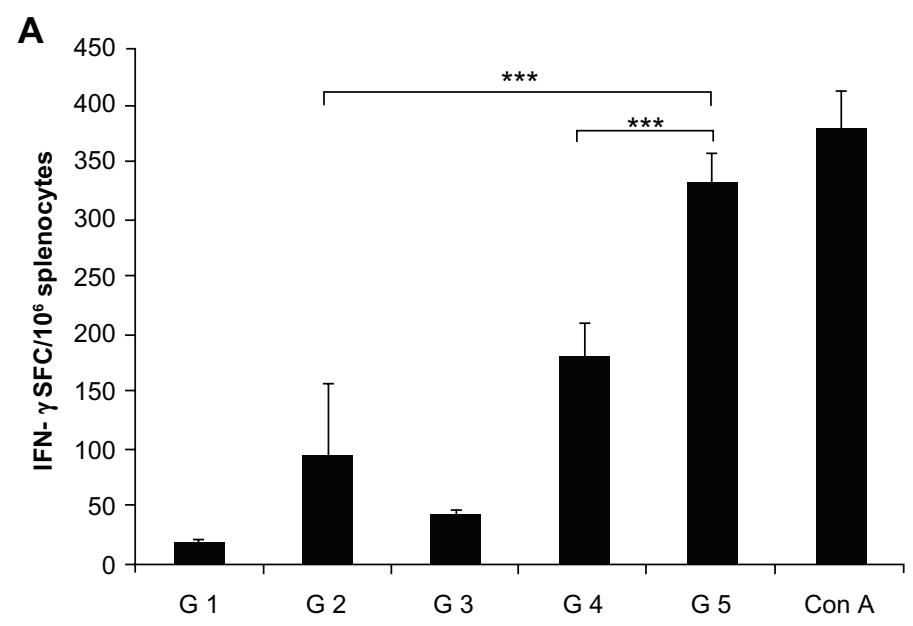

B

(G1)

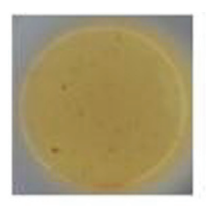

(G4)

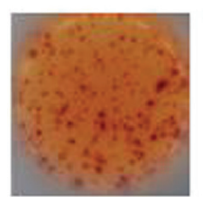

(G2)

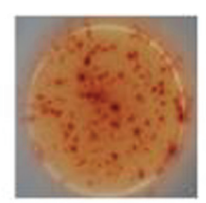

(G5)

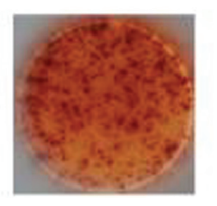

(G3)

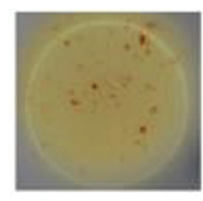

(Con A)

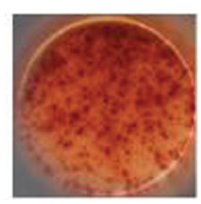

Figure 6 CFP-10 specific IFN- $\gamma$ response to VLP immunization. Splenocytes from different groups of mice were stimulated with CFP- 10 (5 $\mu$ g/mL). Con A ( $5 \mu g / \mathrm{mL})$ was used as control. (A) IFN- $\gamma$-secreting cells detected by ELISPOT assay. (B) Images represent splenic ELISPOT responses.

Notes: The data are expressed as the mean \pm standard deviation. Statistical significance between groups is indicated as $* * * P<0.00 \mathrm{I}$.

Abbreviations: CFP-10, culture filtrate protein-10; ELISPOT, enzyme-linked immunospot; IFN- $\gamma$, interferon-gamma; GI, buffer-immunized group; G2, CFP-I0-immunized group; G3, HBc-VLP-immunized group; G4, HBc + CFP-10-immunized group; G5, fusion protein-immunized group; SFC, spot forming units; Con A, concanavalin A.

with CFP-10 also emphasizes that a Th1 response is actually predominant when Mtb antigen is delivered as an FVLP.

Proliferation analysis indicated that mycobacterial antigen CFP-10 generated significant cell proliferation in mouse splenocytes when antigen was delivered as an FVLP. The ELISPOT analysis also supports the cytokine and ELISA results, indicating the generation of a Th1 immune response in G5 mice.

Adjuvants play a significant role in the nature of immune responses generated by any vaccine and they can direct the immune system in favor of a Th1- or Th2-type response. ${ }^{45,46}$ The Th1 response is essential for protective immunity against intracellular infectious agents and presumably against Mtb also. ${ }^{47}$ The currently available adjuvants, such as water/ oil emulsions and alum, mainly elicit Th2 immunity and are therefore frequently ineffective against intracellular pathogens, such as Mtb, that require a Th1 response. ${ }^{48,49} \mathrm{~A}$ few of the available adjuvants, such as lipid A and its derivatives, are capable of modulating cytokine and IgG isotype profiles characteristic of Th1 immunity. However, they are unable to mount a good T-cell response against soluble or exogenous antigens, a feature that is essential for the development of an effective subunit vaccine against infectious agents like Mtb. ${ }^{45,46,50}$ Functional T cells, especially from a Th1 response, are necessary for generating a protective immune response to $\mathrm{Mtb},{ }^{41,51}$ and therefore for developing an effective TB vaccine, it is essential to identify more vaccine candidates and validate these in animal models.

Recently a study showed the generation of $6 \mathrm{kDa}$ early secretory antigenic target (ESAT-6) specific immune responses in mice after immunization with hepatitis $\mathrm{B}$ core VLPs on $\mathrm{Al}(\mathrm{OH})_{3}$ adjuvant. ${ }^{52}$ This study showed increased antibody responses to ESAT-6 along with an increase in INF- $\gamma$-secreting cells, and our results corroborate their finding that hepatitis B core VLPs can be an efficient antigen carrier. Further, our results strongly suggest that antigen presentation on HBc-VLP obviates the need for an adjuvant and is able to generate Th1 immune responses.

HBc-VLPs are noninfectious. Unlike other VLPs they are not involved in virus receptor interactions and are not a target of neutralizing antibodies, and therefore carrier suppression would not be a major challenge. ${ }^{53,54}$ One of the major constraints in using $\mathrm{HBc}-\mathrm{VLPs}$ as a vaccine delivery system is the inhibition of the assembly of the VLP by the inserted sequence. Therefore, in a modification of an earlier reported work, ${ }^{55}$ we altered the core gene with a Gly-rich linker sequence and inserted restriction sites in the MIR coding region such that foreign protein sequences could be inserted into the modified part. This significantly reduced the problem associated with assembly. HBc-VLPs are generally known to be extremely stable as they are resistant to denaturing agents and variations in $\mathrm{pH}$ and temperature, and these VLPs can be produced in all known homologous and heterologous expression systems. ${ }^{56}$ The capacity to cross present antigens is an added advantage of HBc-VLPs as a vaccine delivery platform against $\mathrm{TB} .{ }^{57,58}$

In conclusion, our results show that the self-adjuvant and immunoenhancing properties of nano-sized $\mathrm{HBc}-$ VLPs could be an excellent platform for vaccine delivery against TB. 


\section{Acknowledgments}

We thank Dr E Sreekumar for valuable suggestions and Sanjay Dharmaseelan and Bindu Asokan for assistance in electron microscopy. DD acknowledges the University Grants Commission for the research fellowship.

\section{Disclosure}

The authors report no conflicts of interest in this work.

\section{References}

1. Dye C, Williams BG. The population dynamics and control of tuberculosis. Science. 2010;328(5980):856-861.

2. Colditz GA, Brewer TF, Berkey CS, et al. Efficacy of BCG vaccine in the prevention of tuberculosis. Meta-analysis of the published literature. JAMA. 1994;271(9):698-702.

3. Rodrigues LC, Diwan VK, Wheeler JG. Protective effect of BCG against tuberculous meningitis and miliary tuberculosis: a meta-analysis. Int $J$ Epidemiol. 1993;22(6):1154-1158.

4. Jouanguy E, Altare F, Lamhamedi S, et al. Interferon-gamma-receptor deficiency in an infant with fatal bacille Calmette-Guerin infection. N Engl J Med. 1996;335(26):1956-1961.

5. Skwarczynski M, Toth I. Peptide-based subunit nanovaccines. Curr Drug Deliv. 2011;8(3):282-289.

6. van Pinxteren LA, Cassidy JP, Smedegaard BH, Agger EM, Andersen P. Control of latent Mycobacterium tuberculosis infection is dependent on CD8 T cells. Eur J Immunol. 2000;30(12):3689-3698.

7. Andersen P, Askgaard D, Ljungqvist L, Bentzon MW, Heron I. T-cell proliferative response to antigens secreted by Mycobacterium tuberculosis. Infect Immun. 1991;59(4):1558-1563.

8. Agger EM, Andersen P. Tuberculosis subunit vaccine development: on the role of interferon-gamma. Vaccine. 2001;19(17-19):2298-2302.

9. Weinrich Olsen A, van Pinxteren LA, Meng Okkels L, Birk Rasmussen P, Andersen P. Protection of mice with a tuberculosis subunit vaccine based on a fusion protein of antigen $85 \mathrm{~b}$ and esat-6. Infect Immun. 2001;69(5):2773-2778.

10. Andersen P. Effective vaccination of mice against Mycobacterium tuberculosis infection with a soluble mixture of secreted mycobacterial proteins. Infect Immun. 1994;62(6):2536-2544.

11. Horwitz MA, Lee BW, Dillon BJ, Harth G. Protective immunity against tuberculosis induced by vaccination with major extracellular proteins of Mycobacterium tuberculosis. Proc Natl Acad Sci U SA. 1995;92(5): $1530-1534$

12. Zhang H, Wen $\mathrm{K}$, Shen J, et al. Characterization of immune responses following intranasal immunization with the Mycobacterium bovis CFP-10 protein expressed by attenuated Salmonella typhimurium. Scand J Immunol. 2010;72(4):277-283.

13. Christy AJ, Dharman K, Dhandapaani G, et al. Epitope based recombinant BCG vaccine elicits specific Th1 polarized immune responses in BALB/c mice. Vaccine. 2012;30(7):1364-1370.

14. Arend SM, Geluk A, van Meijgaarden KE, et al. Antigenic equivalence of human T-cell responses to Mycobacterium tuberculosis-specific RD1-encoded protein antigens ESAT- 6 and culture filtrate protein 10 and to mixtures of synthetic peptides. Infect Immun. 2000;68(6): 3314-3321.

15. Qiao D, Li L, Guo J, et al. Mycobacterium tuberculosis culture filtrate protein 10-specific effector/memory CD4(+) and CD8(+) T cells in tubercular pleural fluid, with biased usage of $\mathrm{T}$ cell receptor Vbeta chains. Infect Immun. 2011;79(8):3358-3365.

16. Aguilar JC, Rodriguez EG. Vaccine adjuvants revisited. Vaccine. 2007;25(19):3752-3762.

17. Hubbell JA, Thomas SN, Swartz MA. Materials engineering for immunomodulation. Nature. 2009;462(7272):449-460.
18. Rice-Ficht AC, Arenas-Gamboa AM, Kahl-McDonagh MM, Ficht TA. Polymeric particles in vaccine delivery. Curr Opin Microbiol. 2010; 13(1):106-112.

19. Grgacic EV, Anderson DA. Virus-like particles: passport to immune recognition. Methods. 2006;40(1):60-65.

20. Bachmann MF, Jennings GT. Vaccine delivery: a matter of size, geometry, kinetics and molecular patterns. Nat Rev Immunol. 2010; 10(11):787-796.

21. Fifis T, Gamvrellis A, Crimeen-Irwin B, et al. Size-dependent immunogenicity: therapeutic and protective properties of nano-vaccines against tumors. J Immunol. 2004;173(5):3148-3154.

22. Bertholet $\mathrm{S}$, Ireton $\mathrm{GC}, \mathrm{Kahn} \mathrm{M}$, et al. Identification of human $\mathrm{T}$ cell antigens for the development of vaccines against Mycobacterium tuberculosis. J Immunol. 2008;181(11):7948-7957.

23. Millington KA, Fortune SM, Low J, et al. Rv3615c is a highly immunodominant RD1 (Region of Difference 1)-dependent secreted antigen specific for Mycobacterium tuberculosis infection. Proc Natl Acad Sci U S A. 2011;108(14):5730-5735.

24. Yu F, Wang J, Dou J, et al. Nanoparticle-based adjuvant for enhanced protective efficacy of DNA vaccine Ag85A-ESAT-6IL-21 against Mycobacterium tuberculosis infection. Nanomedicine. 2012;8(8):1337-1344.

25. Griffiths G, Nystrom B, Sable SB, Khuller GK. Nanobead-based interventions for the treatment and prevention of tuberculosis. Nat Rev Microbiol. 2010;8(11):827-834.

26. Bivas-Benita M, Lin MY, Bal SM, et al. Pulmonary delivery of DNA encoding Mycobacterium tuberculosis latency antigen Rv1733c associated to PLGA-PEI nanoparticles enhances T cell responses in a DNA prime/protein boost vaccination regimen in mice. Vaccine. 2009;27(30):4010-4017.

27. Wizemann H, von Brunn A. Purification of E. coli-expressed HIS-tagged hepatitis B core antigen by Ni2+ -chelate affinity chromatography. J Virol Methods. 1999;77(2):189-197.

28. Vogel M, Diez M, Eisfeld J, Nassal M. In vitro assembly of mosaic hepatitis B virus capsid-like particles (CLPs): rescue into CLPs of assembly-deficient core protein fusions and FRET-suited CLPs. FEBS Lett. 2005;579(23):5211-5216.

29. Hubbard RD, Flory CM, Collins FM. Immunization of mice with mycobacterial culture filtrate proteins. Clin Exp Immunol. 1992;87(1): 94-98.

30. Wu Y, Woodworth JS, Shin DS, Morris S, Behar SM. Vaccine-elicited 10-kilodalton culture filtrate protein-specific CD8+T cells are sufficient to mediate protection against Mycobacterium tuberculosis infection. Infect Immun. 2008;76(5):2249-2255.

31. Stukova MA, Sereinig S, Zabolotnyh NV, et al. Vaccine potential of influenza vectors expressing Mycobacterium tuberculosis ESAT-6 protein. Tuberculosis (Edinb). 2006;86(3-4):236-246.

32. Orme IM, Andersen P, Boom WH. T cell response to Mycobacterium tuberculosis. J Infect Dis. 1993;167(6):1481-1497.

33. Casadevall A. Antibody-mediated immunity against intracellular pathogens: two-dimensional thinking comes full circle. Infect Immun. 2003;71(8):4225-4228.

34. Williams A, Reljic R, Naylor I, et al. Passive protection with immunoglobulin A antibodies against tuberculous early infection of the lungs. Immunology. 2004;111(3):328-333.

35. Finkelman FD, Holmes J, Katona IM, et al. Lymphokine control of in vivo immunoglobulin isotype selection. Апnu Rev Immunol. 1990;8: 303-333.

36. Natsuume-Sakai S, Motonishi K, Migita S. Quantitative estimations of five classes of immunoglobulin in inbred mouse strains. Immunology. 1977;32(6):861-866.

37. Flynn JL, Chan J, Triebold KJ, Dalton DK, Stewart TA, Bloom BR. An essential role for interferon gamma in resistance to Mycobacterium tuberculosis infection. J Exp Med. 1993;178(6):2249-2254.

38. Smith KA. Interleukin-2: inception, impact, and implications. Science. 1988;240(4856):1169-1176. 
39. Zhang M, Lin Y, Iyer DV, Gong J, Abrams JS, Barnes PF. T-cell cytokine responses in human infection with Mycobacterium tuberculosis. Infect Immun. 1995;63(8):3231-3234.

40. Young S, O’Donnell M, Lockhart E, et al. Manipulation of immune responses to Mycobacterium bovis by vaccination with IL-2- and IL-18-secreting recombinant bacillus Calmette Guerin. Immunol Cell Biol. 2002;80(3):209-215.

41. Flynn JL, Goldstein MM, Triebold KJ, Koller B, Bloom BR. Major histocompatibility complex class I-restricted $\mathrm{T}$ cells are required for resistance to Mycobacterium tuberculosis infection. Proc Natl Acad Sci U S A. 1992;89(24):12013-12017.

42. Chen CY, Huang D, Wang RC, et al. A critical role for CD8 T cells in a nonhuman primate model of tuberculosis. PLoS Pathog. 2009;5(4): e1000392.

43. Bogdan C, Moll H, Solbach W, Rollinghoff M. Tumor necrosis factor-alpha in combination with interferon-gamma, but not with interleukin 4 activates murine macrophages for elimination of Leishmania major amastigotes. Eur J Immunol. 1990;20(5):1131-1135.

44. Bekker LG, Freeman S, Murray PJ, Ryffel B, Kaplan G. TNF-alpha controls intracellular mycobacterial growth by both inducible nitric oxide synthase-dependent and inducible nitric oxide synthase-independent pathways. J Immunol. 2001;166(11):6728-6734.

45. Gupta RK. Aluminum compounds as vaccine adjuvants. Adv Drug Deliv Rev. 1998;32(3):155-172.

46. Sun HX, Qin F, Ye YP. Relationship between haemolytic and adjuvant activity and structure of protopanaxadiol-type saponins from the roots of Panax notoginseng. Vaccine. 2005;23(48-49):5533-5542.

47. McMurray DN. Determinants of vaccine-induced resistance in animal models of pulmonary tuberculosis. Scand J Infect Dis. 2001;33(3): 175-178.

48. Petrovsky N. Novel human polysaccharide adjuvants with dual Th1 and Th2 potentiating activity. Vaccine. 2006;24 Supp1 2:S2-26-29.

49. Yang X, Bao L, Deng Y. A novel recombinant Mycobacterium bovis bacillus Calmette-Guerin strain expressing human granulocyte macrophage colony-stimulating factor and Mycobacterium tuberculosis early secretory antigenic target 6 complex augments Th1 immunity. Acta Biochim Biophys Sin (Shanghai). 2011;43(7):511-518.
50. Soltysik S, Wu JY, Recchia J, et al. Structure/function studies of QS-21 adjuvant: assessment of triterpene aldehyde and glucuronic acid roles in adjuvant function. Vaccine. 1995;13(15):1403-1410.

51. Wangoo A, Sparer T, Brown IN, et al. Contribution of Th1 and Th2 cells to protection and pathology in experimental models of granulomatous lung disease. J Immunol. 2001;166(5):3432-3439.

52. Yin Y, Li H, Wu S, et al. Hepatitis B virus core particles displaying Mycobacterium tuberculosis antigen ESAT-6 enhance ESAT-6-specific immune responses. Vaccine. 2011;29(34):5645-5651.

53. Pumpens P, Grens E. Hepatitis B core particles as a universal display model: a structure-function basis for development. FEBS Lett. 1999;442(1):1-6.

54. Ruedl C, Schwarz K, Jegerlehner A, Storni T, Manolova V, Bachmann MF. Virus-like particles as carriers for T-cell epitopes: limited inhibition of T-cell priming by carrier-specific antibodies. JVirol. 2005;79(2):717-724.

55. Kratz PA, Bottcher B, Nassal M. Native display of complete foreign protein domains on the surface of hepatitis B virus capsids. Proc Natl Acad Sci U S A. 1999;96(5):1915-1920.

56. Newman M, Suk FM, Cajimat M, Chua PK, Shih C. Stability and morphology comparisons of self-assembled virus-like particles from wild-type and mutant human hepatitis B virus capsid proteins. JVirol. 2003;77(24):12950-12960.

57. Ruedl C, Storni T, Lechner F, Bachi T, Bachmann MF. Cross-presentation of virus-like particles by skin-derived CD8(-) dendritic cells: a dispensable role for TAP. Eur J Immunol. 2002;32(3):818-825.

58. Jegerlehner A, Tissot A, Lechner F, et al. A molecular assembly system that renders antigens of choice highly repetitive for induction of protective B cell responses. Vaccine. 2002;20(25-26):3104-3112.
International Journal of Nanomedicine

\section{Publish your work in this journal}

The International Journal of Nanomedicine is an international, peerreviewed journal focusing on the application of nanotechnology in diagnostics, therapeutics, and drug delivery systems throughout the biomedical field. This journal is indexed on PubMed Central,

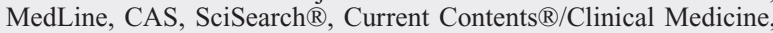

\section{Dovepress}

Journal Citation Reports/Science Edition, EMBase, Scopus and the Elsevier Bibliographic databases. The manuscript management system is completely online and includes a very quick and fair peer-review system, which is all easy to use. Visit http://www.dovepress.com/ testimonials.php to read real quotes from published authors. 